\title{
Polarons and bipolarons in strongly interacting electron-phonon systems
}

\author{
G. Wellein, H. RÖDER And H. Fehske \\ Physikalisches Institut, Universität Bayreuth, D-95440 Bayreuth, Germany \\ (Bayreuth, December 19, 1995)
}

\begin{abstract}
The Holstein Hubbard and Holstein $\mathrm{t}-\mathrm{J}$ models are studied for a wide range of phonon frequencies, electron-electron and electron-phonon interaction strengths on finite lattices with up to ten sites by means of direct Lanczos diagonalization. Previously the necessary truncation of the phononic Hilbert space caused serious limitations to either very small systems (four or even two sites) or to weak electron-phonon coupling, in particular in the adiabatic regime. Using parallel computers we were able to investigate the transition from 'large' to 'small' polarons in detail. By resolving the low-lying eigenstates of the Hamiltonian and by calculating the spectral function we can identify a polaron band in the strong-coupling case, whose dispersion deviates from the free-particle dispersion at low and intermediate phonon frequencies. For two electrons (holes) we establish the existence of bipolaronic states and discuss the formation of a bipolaron band. For the 2D Holstein t-J model we demonstrate that the formation of hole-polarons is favoured by strong Coulomb correlations. Analyzing the hole-hole correlation functions we find that hole binding is enhanced as a dynamical effect of the electron-phonon interaction.
\end{abstract}


PACS number(s):: 71.27.+a, 71.38.+i, 74.25.Kc, 75.10.Lp 


\section{INTRODUCTION}

Following the discovery of high-temperature superconductivity in the ceramic copper oxides, novel purely electronic pairing mechanisms due to the strong Coulomb correlations within the $\mathrm{CuO}_{2}$ planes have been investigated in detail. Recently, however, it has become clear that the lattice degrees of freedom are essential in understanding the puzzling normalstate properties of the cuprates [1] 3]. Even if it should turn out that the electron-phonon (EP) interaction is not the relevant pairing interaction in those materials, its effects need to be reconsidered for the case of strong electron-electron interactions and low effective dimensionality as realized in the high- $T_{c}$ superconductors. In particular, polaronic effects are suggested to play a non-negligible role in the copper-based materials $\mathrm{La}_{2-\mathrm{x}} \mathrm{Sr}_{\mathrm{x}} \mathrm{CuO}_{4+\mathrm{y}}$ 四 9 and even more in the isostructural nickel-based charge-transfer oxides $\mathrm{La}_{2-\mathrm{x}} \mathrm{Sr}_{\mathrm{x}} \mathrm{NiO}_{4+\mathrm{y}}$ [7],10]. Experimentally, photo-induced absorption experiments [11], infrared spectroscopy [12] as well as infrared reflectivity measurements [13 unambiguously indicate the formation of 'selflocalized' polaronic states (small polarons) in the insulating parent compounds $\mathrm{La}_{2} \mathrm{CuO}_{4+\mathrm{y}}$ and $\mathrm{Nd}_{2} \mathrm{CuO}_{4-\mathrm{y}}$ of the hole- and electron-doped superconductors $\mathrm{La}_{2-\mathrm{x}} \mathrm{Sr}_{\mathrm{x}} \mathrm{CuO}_{4+\mathrm{y}}$ and $\mathrm{Nd}_{2-\mathrm{x}} \mathrm{Ce}_{\mathrm{x}} \mathrm{CuO}_{4-\mathrm{y}}$, respectively. Therefore a growing theoretical interest in the study of strongly correlated EP models can be found in the recent literature [14 26].

Probably the simplest microscopic models including both the electron and phonon degrees of freedom are the Holstein Hubbard model

$$
\begin{aligned}
\mathcal{H}_{H-H}=-t & \sum_{\langle i j\rangle \sigma}\left(c_{i \sigma}^{\dagger} c_{j \sigma}+\text { H.c. }\right)+U \sum_{i} n_{i \uparrow} n_{i \downarrow} \\
& -\sqrt{\varepsilon_{p} \hbar \omega} \sum_{i}\left(b_{i}^{\dagger}+b_{i}\right) n_{i}+\hbar \omega \sum_{i}\left(b_{i}^{\dagger} b_{i}+\frac{1}{2}\right)
\end{aligned}
$$

and the Holstein $\mathrm{t}-\mathrm{J}$ model

$$
\begin{array}{r}
\mathcal{H}_{H-t-J}=-t \sum_{\langle i j\rangle \sigma}\left(\tilde{c}_{i \sigma}^{\dagger} \tilde{c}_{j \sigma}+\text { H.c. }\right)+J \sum_{\langle i j\rangle}\left(\vec{S}_{i} \vec{S}_{j}-\frac{1}{4} \tilde{n}_{i} \tilde{n}_{j}\right) \\
-\sqrt{\varepsilon_{p} \hbar \omega} \sum_{i}\left(b_{i}^{\dagger}+b_{i}\right) \tilde{h}_{i}+\hbar \omega \sum_{i}\left(b_{i}^{\dagger} b_{i}+\frac{1}{2}\right),
\end{array}
$$


where $c_{i \sigma}^{(\dagger)}$ annihilates (creates) an electron at Wannier site $i$ with spin projection $\sigma$, $n_{i}=n_{i \uparrow}+n_{i \downarrow}$, and $t$ denotes the transfer amplitude between nearest-neighbour (NN) pairs $\langle i j\rangle . \mathcal{H}_{H-t-J}$ acts in a projected Hilbert space without double occupancy, i.e., $\tilde{c}_{i \sigma}^{(\dagger)}=c_{i \sigma}^{(\dagger)}\left(1-n_{i \bar{\sigma}}\right)$, and $\vec{S}_{i}=\frac{1}{2} \sum_{\sigma \sigma^{\prime}} \tilde{c}_{i \sigma}^{\dagger} \vec{\tau}_{\sigma \sigma^{\prime}} \tilde{c}_{i \sigma^{\prime}}$. The first two terms in (1) and (2) represent the standard Hubbard model and t- $\mathrm{J}$ model, respectively, where $U$ is the on-site Coulomb repulsion and $J$ measures the NN antiferromagnetic exchange interaction strength. The third and fourth terms take into account the EP interaction and the phonon energy in a harmonic approximation. Here, the on-site electron (hole) occupation number $n_{i}\left(\tilde{h}_{i}=1-\tilde{n}_{i}\right)$ is locally coupled to a dispersionsless optical phonon mode, where $\varepsilon_{p}$ is the EP coupling constant, $\omega$ denotes the bare phonon frequency, and $b_{i}^{(\dagger)}$ are the phonon annihilation (creation) operators. In the context of an effective single-band description of the copper/nickel oxides, the collective Holstein-coordinates $q_{i}=\sqrt{\hbar / 2 M \omega}\left(b_{i}^{\dagger}+b_{i}\right)$ may be thought of as representing an internal optical degree of freedom of the lattice site $i$, i.e., in this case the dominant source of EP coupling is assumed to result from the interaction of dopant-induced charge carriers with the apical out-of plane or the bond-parallel in-plane breathing-type displacements of oxygen atoms.

Unfortunately, for strongly coupled EP systems exact results exist only in a few special cases and limits 27 30]. Whereas, in an approximative treatment, the weak-coupling regime $\left(\varepsilon_{p} / t \ll 1\right)$ is well understood and dealt with by perturbation theory, the standard strong-coupling Migdal-Eliashberg theory [31,32 based on the adiabatic Migdal theorem might break down for strong enough EP interactions $\left(\varepsilon_{p} / t \gg 1\right)$ due to the familiar polaronic band collapse [5]. Note that in the presence of strong Coulomb correlations, a rather moderate EP can cause a substantial reduction of the coherent band motion making the particles susceptible to 'self-trapping' [16,24]. The (single) polaron problem has been tackled in the strong-coupling adiabatic $(\hbar \omega / t \ll 1)$ and antiadiabatic $(\hbar \omega / t \gg 1)$ limits using the Holstein [33] and Lang-Firsov [34] approximations, respectively. Both approaches yield a narrow polaronic band with an exponentially reduced half-bandwidth [18]. Whether these 
small polarons (or bipolarons) can exist as itinerant band states is still a heavily debated issue [1]. Apart from variational calculations [35 37,20 little is known for intermediate values of EP coupling and phonon frequency $\varepsilon_{p} \sim \hbar \omega \sim t$ and, in particular, for the manypolaron problem. In principle, exact diagonalization (ED) 14,38, 18, 26, and (quantum) Monte Carlo [39 43] methods including the full quantum nature of phonons can close this gap. However, by using direct ED techniques it is necessary to truncate the phononic Hilbert space, and hence the accessible parameter space is limited by the size of the matrix one can diagonalize. Therefore ED studies up to now were limited to either small values of $\varepsilon_{p}$, to the so-called frozen phonon approximation [44 47, or to very small systems [14, 15, 18, 26]. In a previous work [24], the authors have proposed a variational Lanczos diagonalization technique on the basis of an inhomogeneous modified variational Lang-Firsov transformation (IMVLF) that allows for the description of static displacement field, polaron and squeezing effects in terms of the Holstein $\mathrm{t}-\mathrm{J}$ and Holstein Hubbard models on fairly large clusters. Although the adiabatic and antiadiabatic as well as the weak- and strong-coupling limiting cases are well reproduced in this approach, the situation becomes less favourable at intermediate EP couplings and phonon frequencies and, in particular, in the crossover region from large-size nearly free polarons (FP) to small-size 'quasi-localized' polarons (i.e., in the vicinity of the so-called 'self-trapping' transition). Obviously, this regime requires a more accurate treatment of the phonons as quantum mechanical objects.

Encouraged by this situation it is the aim of the present paper to perform a direct Lanczos diagonalization of the Holstein Hubbard and Holstein t-J models, preserving the full dynamics of quantum phonons. In particular, we investigate for the first time the low-lying excitations (spectral functions) on large enough lattices, in order to identify the dispersion relation of the (bi)polaronic quasiparticles. 


\section{COMPUTATIONAL PROCEDURE}

A general state of the model Hamiltonian $\mathcal{H}_{H-H}\left[\mathcal{H}_{H-t-J}\right]$ describing $N_{e l}=N_{\uparrow}+N_{\downarrow}$ electrons on a finite $D$-dimensional hypercubic lattice with $N$ sites can be written as the direct product

$$
|\Psi\rangle=\sum_{l, k} c_{l}^{k}|l\rangle_{e l} \otimes|k\rangle_{p h}
$$

where $l$ and $k$ label the basic states of the electronic and phononic Hilbert space with dimensions $D_{e l}=\left(\begin{array}{c}N \\ N_{\uparrow}\end{array}\right)\left(\begin{array}{c}N \\ N_{\downarrow}\end{array}\right)\left[D_{e l}=\left(\begin{array}{c}N \\ N_{\uparrow}\end{array}\right)\left(\begin{array}{c}N-N_{\uparrow} \\ N_{\downarrow}\end{array}\right)\right]$ and $D_{p h}=\infty$, respectively. Since the bosonic part of the Hilbert space is infinite dimensional we use a truncation procedure 48 restricting ourselves to phononic states with at most $M$ phonons:

$$
|k\rangle_{p h}=\prod_{i=1}^{N} \frac{1}{\sqrt{n_{i}^{k} !}}\left(b_{i}^{\dagger}\right)^{n_{i}^{k}}|0\rangle_{p h}
$$

with

$$
\sum_{i=1}^{N} n_{i}^{k} \leq M
$$

and $1 \leq k \leq D_{p h}^{(M)}=(M+N) ! / M ! N$ !. To further reduce the dimension of the Hilbert space, in the case of $\mathcal{H}_{H-H}$ we separate out the center of mass motion by transforming

to new phonon operators $B_{i}^{(\dagger)}$, which can be taken into account analytically as displaced harmonic oscillators. For the Holstein $\mathrm{t}-\mathrm{J}$ model it is more effective to exploit the point group symmetries of the original basis (3).

Then the resulting Hamiltonian matrix is diagonalized using a standard Lanczos method. As the convergence of the Lanczos procedure depends on the (relative) difference of neighbouring eigenvalues, $\left|E_{i+1}-E_{i}\right| /\left|E_{i}\right|$, one needs to be very careful in resolving eigenvalues within the extremely narrow small-polaron band. To monitor the convergence of our truncation procedure as a function of $M$ we calculate the weight of the $m$-phonon states in the ground state $\left|\Psi_{0}\right\rangle$ of $\mathcal{H}$ :

$$
\left|c^{m}\right|^{2}=\sum_{l, k}\left|c_{l}^{k}\right|^{2}, \quad \text { where } m=\sum_{i=1}^{N} n_{i}^{k} .
$$


At fixed $M$, the curve $\left|c^{m}\right|^{2}(m)$ is bell-shaped and its maximum corresponds to the most probable number of phonon quanta in the ground state. To illustrate the $M$-dependences of the ground-state energy $E_{0}$ and the coefficients $\left|c^{m}\right|^{2}$, we have shown both quantities for the single-electron Holstein model in Fig. 1. In the numerical work convergence is achieved if the relative error of the ground-state energy is less than $10^{-7}$. In addition, we check that $E_{0}$ is smaller than the estimate obtained from the IMVLF-Lanczos treatment of the phonon subsystem [24].

We have written the program in Fortran90 and ran it on a 64-node CM5. We were able to diagonalize Hamiltonian matrices up to a total dimension $\left(D_{t o t}\right)$ of about 82 millions. Since a matrix vector multiplication for this matrix size takes less than 150 seconds, the limiting factor of our numerical algorithm is the available storage.

\section{NUMERICAL RESULTS}

\section{A. Holstein Hubbard model}

\section{One-electron case}

In the first place, we investigate the polaron properties of the Holstein model with a single electron on finite lattices with up to ten sites using periodic boundary conditions. In the light of the literature over at least the last two decades [49, 39, 26, 28, 30] we expect a gradual transition from a (nearly free) large-polaron solution to a small-polaron-like ground state upon increasing the EP coupling. Since, in particular in the adiabatic regime, the formation of a polaronic state is accompanied by a strong reduction of the coherent electron motion, this effect should be observable in the expectation value of the kinetic energy $E_{p, k i n} / t=-\sum_{<i j>\sigma}\left\langle\Psi_{0}\right|\left(c_{i \sigma}^{\dagger} c_{j \sigma}+\right.$ H.c. $)\left|\Psi_{0}\right\rangle$, where $\left|\Psi_{0}\right\rangle$ is the ground-state wavefunction. We therefore define an effective polaronic transfer amplitude [24],

$$
t_{p, e f f}=E_{p, k i n}\left(\varepsilon_{p}, U\right) / E_{p, k i n}(0, U),
$$


in order to characterize the increase in the quasiparticle mass [24]. Note that $t_{p, e f f}$ substantially differs from the (exponential) polaron band renormalization factor $(\rho)$ obtained analytically in the non-adiabatic Lang-Firsov and adiabatic Holstein cases [18.

We illustrate the dependence of this effective hopping amplitude on the EP interaction strength in Fig. 2, where we have plotted $t_{p, e f f}$ as a function of $\varepsilon_{p}$ at different phonon frequencies. First it is important to realize that there are two complementary (adiabatic and non-adiabatic) regimes for the polaronic motion. In the non-adiabatic regime, where the lattice fluctuations are fast and the phonons are able to follow immediately the electronic motion forming a non-adiabatic Lang-Firsov polaron (NLFP), one observes a very gradual decrease of $t_{p, \text { eff }}$ as $\varepsilon_{p}$ increases. At the same time the 'phonon distribution function', $\left|c^{m}\right|^{2}$, gets wider but the maximum is still located at the zero-phonon state. In the adiabatic regime, one notices a crossover from a large-size polaron (LP) in 1D or nearly free polaron $(\mathrm{FP})$ in $2 \mathrm{D}$, described by a $t_{p, e f f}$ that is only weakly reduced from its noninteracting value, to a less mobile (small-size) adiabatic Holstein polaron (AHP) for large $\varepsilon_{p}$. We point out that the nature of 'delocalized' polaronic states, occurring in the weak-coupling region, is different in $1 \mathrm{D}$ and $2 \mathrm{D}$ [24]. In the $1 \mathrm{D}$ case, the FP state becomes unstable at any finite EP coupling. As expected the transition to the AHP state occurs if the EP coupling approximately exceeds half the bare electronic bandwidth and, in accordance with Monte Carlo results [39,40, is much sharper in two dimensions [51] (in the remainder of this section we focus on the $1 \mathrm{D}$ case). Nonetheless, all physical quantities are smooth functions of $\varepsilon_{p}$, in particular there are no ground-state level crossings, i.e., the transition from LP/FP to AHP is continuous and not accompanied by any non-analyticities. While in the weakcoupling case we have $m_{\max }=0$ and the inclusion of higher phonon states $(m \gtrsim 5)$ does not improve the ground-state energy at all, in the adiabatic strong-coupling case $\left(\varepsilon_{p}=4\right.$, $\hbar \omega=0.4)$, the maximum in $\left|c^{m}\right|^{2}$ is shifted to multi-phonon states $\left(m_{\max } \simeq 8\right)$ and we need about 16 phonons to reach a sufficient accuracy within our truncation procedure. Note that a similar behaviour can be observed in the non-adiabatic regime $(\hbar \omega>t)$ provided that $\varepsilon_{p} \gg \hbar \omega$, e.g., for $\hbar \omega=3$ and $\varepsilon_{p}=8\left(\varepsilon_{p}=10\right)$ we find $m_{\max } \simeq 2$ in $1 \mathrm{D}$ (2D). These results 
confirm previous findings for the Holstein Hubbard model on very small size clusters (with two or three sites), where, as $\varepsilon_{p}$ increases in the adiabatic regime, a strong increase of the average number of phonons, $\left\langle N_{p h}\right\rangle$, contained in the ground state, was observed (cf. Tab. I in Ref. [14 and Tab. I in Ref. [15]). In the center of mass system, the phonon expectation value in the polaronic ground state may be derived from the phonon distribution function $\left|c^{m}\right|^{2}$ by $\left\langle N_{p h}\right\rangle=\sum_{m=0}^{M}\left|c^{m}\right|^{2}+\frac{\varepsilon_{p}}{\hbar \omega} \frac{N_{e l}^{2}}{N}$.

To elucidate the difference between the 'extended' LP and 'quasi-localized' AHP states in more detail, we have calculated the electron-phonon density correlation function

$$
C_{e l-p h}(|i-j|)=\left\langle\Psi_{0}\left|n_{i} b_{j}^{\dagger} b_{j}\right| \Psi_{0}\right\rangle
$$

which measures the correlation between the electron occupying site $i$ and the density of phonons on site $j$ [50]. Results for $C_{e l-p h}(|i-j|)$, plotted in Fig. 3 at $\hbar \omega=0.4$ for all distances $i-j:=\vec{R}_{i}-\vec{R}_{j}$, show that for small $\varepsilon_{p}$ the correlation between the electron and the phonons is pretty weak and exhibits little structure, i.e., the few phonons contained in the ground state are nearly uniformly distributed over the whole lattice. In contrast, in the case of large EP coupling $\left(\varepsilon_{p}=3\right)$, the phonons are strongly correlated with the position of the electron, thus implying a very small radius of the polaron. Note, however, that the translational invariance of the ground state is not broken. Since a polaron's mass is inversely proportional to its size, the AHP formed at large $\varepsilon_{p}$ is an extremely heavy quasiparticle. As can be seen from the inset of Fig. 3, the on-site electron-phonon correlation increases dramatically around the same value of $\varepsilon_{p}$ at which $t_{p, \text { eff }}$ becomes depressed (cf. Fig. 2). This means, in the adiabatic regime a strong short-range EP interaction can lower the energy of the system due to a deformation-potential-like contribution sufficiently to overcompensate the loss of kinetic energy. Nonetheless, the 'quasi-localized' (self-trapped) polaronic state has band-like character, i.e., the AHP can move itinerantly.

In order to discuss the formation of a small-polaron band one has to calculate the low-lying excited states. As a first step, in Fig. Twe classify the lowest eigenvalues of the Holstein model according to the allowed wave-vectors of the eight-site lattice for various 
phonon frequencies at $\varepsilon_{p}=3$. Here the 'band dispersion' $E_{K}-E_{0}$ is scaled with respect to the so-called coherent bandwidth $\Delta E=\sup _{K} E_{K}-\inf _{K} E_{K} . \Delta E$ strongly depends on both ratios $\varepsilon_{p} / \hbar \omega$ and $\varepsilon_{p} / t$, for example, we found $\Delta E\left(\varepsilon_{p}=3, \hbar \omega\right)=0.0157,0.1957,2.9165$, and 4.0 for $\hbar \omega=0.4,0.8,10.0$ and $\infty$, respectively. Of course, the simple Lang-Firsov formula, $\Delta E_{L F}=4 \mathrm{D} \exp \left[-\varepsilon_{p} / \hbar \omega\right]$, gives a good estimate of the polaronic bandwidth only in the nonadiabatic regime: $\Delta E_{L F}\left(\varepsilon_{p}=3, \hbar \omega\right)=0.0022(\hbar \omega=0.4), 0.0941(0.8), 2.9633(10.0), 4(\infty)$. Besides the strong renormalization of the bandwidth in the low-frequency strong-coupling regime it is interesting to note that the deviation of the polaron band dispersion from a (rescaled) 'cosine-dispersion' of noninteracting electrons is most pronounced at intermediate phonon frequencies $\hbar \omega \sim t$, i.e., in between the extreme adiabatic (AHP) and antiadiabatic (NLFP) limits. This deviation may be due to a residual polaron-phonon interaction, with the phonons sitting on sites other than the polaron. To demonstrate that the low-lying eigenvalues do indeed form a well-separated quasiparticle band in the adiabatic strongcoupling regime $\left(\varepsilon_{p}=3, \hbar \omega=0.4\right)$, in the inset of Fig. $\frac{1}{4}$ we have displayed the lowest few eigenvalues in dependence on $\varepsilon_{p}$. In the very weak-coupling regime $\left(\varepsilon_{p}=0.5\right)$ the eigenvalues are barely changed from their $\varepsilon_{p}=0$ values, where additional eigenvalues, separated from the ground-state energy $E_{0}$ by multiples of $\hbar \omega$ (e.g., $E_{2}, E_{3}$, and $E_{4}$ ), enter the spectrum. As $\varepsilon_{p}$ increases a band of states separates from the rest of the spectrum. These states become very close in energy and a narrow well-separated energy band evolves in the strong-coupling case $\left(\varepsilon_{p}=3\right)$. Obviously, the gap to the next higher band of eigenvalues is of the order of the bare phonon frequency $\hbar \omega$. Neglecting degeneracies one may tentatively identify those five states as the states of the small-polaron band on the eight-site lattice.

Keeping this identification in mind, in Fig. 5 we have plotted the lowest eigenvalues as a function of the (1D) $K$-vectors belonging to various system sizes $(N=6,8,10)$. One notices that the dispersion $E_{K}$ is rather size independent, i.e., the $E_{K}$ values obtained for larger systems just fill the gaps. Undoubtedly, the smooth shape of $E_{K}$ already provides good reasons for a quasiparticle band description of the AHP in the strong-coupling regime. To further substantiate this quasiparticle interpretation, we also have calculated the one- 
particle spectral functions

$$
A_{K}(E)=\sum_{n}\left|\left\langle\Psi_{n}^{\left(N_{e l}\right)}\left|c_{K}^{\dagger}\right| \Psi_{0}^{\left(N_{e l}-1\right)}\right\rangle\right|^{2} \delta\left(E-E_{n}^{\left(N_{e l}\right)}+E_{0}^{\left(N_{e l}-1\right)}\right)
$$

with $N_{e l}=1$ for the non-equivalent $K$-values of the six-site system using a polynomial moment method [52]. The idea is to see a direct verification of the coherent band dispersion $E_{K}$ in terms of $A_{K}(E)$. The electronic spectral functions $A_{K}(E)$ are shown in the four insets of Fig. 5. The important point, we would like to emphasize, is that the position of the first peak in each spectral function $A_{K}(E)$ exactly coincides with the corresponding $E_{K}$-value and the other peaks are at higher energies than any of the coherent band-energy values. This means, our exact results for the low-energy excitation spectrum of a single electron corroborate the existence of heavily dressed polaronic quasiparticles, where the electronic and phononic degrees of freedom are strongly mixed. Of course, in the very high-energy regime the results for $A_{K}(E)$ can not be trusted just due to the errors induced by the necessary truncation of the phononic Hilbert space.

\section{Two-electron case}

Next, we wish to discuss the two-electron problem. Here it is of special interest to understand in detail the conditions under which the two electrons form a bipolaron. Whether or not a transition to a bipolaronic state will occur depends sensitively on the competition between the short-ranged phonon-mediated, i.e., retarded $(\hbar \omega<\infty)$, attraction $\left(\propto \varepsilon_{p}\right)$ and the instantaneous on-site Hubbard repulsion $(\propto U)$.

We start again with a discussion of the mobility of the particles. Fig. 6 (a) shows the strong (gradual) reduction of the effective polaronic transfer amplitude $t_{p, \text { eff }}$ as $\varepsilon_{p}$ increases in the adiabatic (non-adiabatic) regime. Now let us mainly focus on the physically more interesting regime of 'small' phonon frequencies, $\hbar \omega=0.4$. In the case of vanishing Coulomb interaction $U=0$ any finite EP interaction causes an effective on-site attraction between the electrons forming a bipolaronic bound state (remember, e.g., that $U_{\text {eff }}=U-2 \varepsilon_{p}$ follows 
from the simple Lang-Firsov approach). This means, in the pure Holstein model the state with two nearly free (large) polarons does not exist, at least in one spatial dimension [14. In the weak-coupling limit, the two-polaron state can, however, be stabilized by taking into account the on-site Coulomb repulsion. In this case, a crossover from a state of two mobile large polarons to an extended bipolaronic state occurs. The 'transition' will be shifted to larger EP couplings as $U$ increases (see Fig. 6 (a)). For example, at $U=6$ and $\hbar \omega=0.4$ (3.0), we find that the binding energy of two electrons, $E_{B}^{2}=E_{0}(2)-2 E_{0}(1)$, becomes negative at about $\varepsilon_{p}=1.7(2.8)$. Further justification for this interpretation can be found from the behaviour of the effective bipolaronic transfer amplitude [53],

$$
t_{b, e f f}=E_{b, k i n}\left(\varepsilon_{p}, U\right) / E_{b, k i n}(0, U)
$$

with

$$
E_{b, k i n}\left(\varepsilon_{p}, U\right) / t=-\sum_{<i j\rangle}\left\langle\Psi_{0}\left(\varepsilon_{p}, U\right)\right|\left(c_{i \uparrow}^{\dagger} c_{i \downarrow}^{\dagger} c_{j \uparrow} c_{j \downarrow}+\text { H.c. }\right)\left|\Psi_{0}\left(\varepsilon_{p}, U\right)\right\rangle,
$$

shown in Fig. (6) (b). $t_{b, e f f}$ describes the coherent hopping of a on-site bipolaron from site $i$ to site $j$. Contrary to $t_{p, e f f}$, at low EP coupling strengths, the bipolaronic hopping amplitude $t_{b, e f f}$ grows with increasing $\varepsilon_{p}$ showing the increasing importance of the correlated motion of two electrons (but, quite clearly, we have $\left|E_{b, k i n}\right|<\left|E_{p, k i n}\right|$ ). At large EP couplings (e.g., for $\varepsilon_{p} \gtrsim 1$ at $U=0$ and $\hbar \omega=0.4$ ), the on-site bipolaron becomes more and more localized and accordingly we observe a drop in $t_{b, e f f}$ which corresponds to the drop in $t_{p, e f f}$ in the case of one electron at the parameter values where the AHP becomes stable. Hence we will call this quasiparticle an adiabatic Holstein bipolaron (AHBP).

To better illustrate the effect of pair formation in the 1D Holstein (Hubbard) model, we present in Fig. 7 the electron-electron density correlation function

$$
C_{e l-e l}(|i-j|)=\left\langle\Psi_{0}\left(\varepsilon_{p}, U\right)\left|n_{i} n_{j}\right| \Psi_{0}\left(\varepsilon_{p}, U\right)\right\rangle-\left\langle\Psi_{0}(0, U)\left|n_{i} n_{j}\right| \Psi_{0}(0, U)\right\rangle
$$

in the adiabatic regime with (b) and without (a) Hubbard repulsion. In each case we have displayed the results for $C_{e l-e l}(|i-j|)$ as a function of $\varepsilon_{p}$ in comparison to the electronphonon correlation function $C_{e l-p h}(|i-j|)$ given by (8). As Fig. (7) (a) shows, in the limit of 
vanishing Coulomb interaction the on-site electron-electron correlation $C_{e l-e l}(0)$ dominates the inter-site correlations $C_{e l-e l}(|i-j|)$ with $|i-j| \geq 1$, in particular for $\varepsilon_{p} \gtrsim 0.9$, i.e., in the AHBP regime where both electrons are mainly confined to the same site sharing a common lattice distortion. Therefore the transition from a mobile large bipolaron to a 'quasi-self-trapped' on-site AHBP is manifest in a strongly enhanced $C_{e l-p h}(0)$ as well (see inset). Moreover, the transition should be associated with a significant reduction of the local magnetic moment, $m_{l o c}\left(\varepsilon_{p}, U\right) \propto\left\langle\Psi_{0}\left|\left(n_{i \uparrow}-n_{i \downarrow}\right)^{2}\right| \Psi_{0}\right\rangle$, indicating the local pairing of spin up and down electrons. Indeed we found $m_{l o c}\left(\varepsilon_{p}=1\right) /\left.m_{l o c}\left(\varepsilon_{p}=0.9\right)\right|_{U=0, \hbar \omega=0.4}=0.66$. As can be seen from Fig. 7 (b), a somewhat different scenario emerges in the presence of a finite Coulomb interaction. Here, the Hubbard repulsion prevents the formation of an on-site bipolaronic bound state in the weak EP coupling regime. On the other hand, as recently pointed out by Marsiglio [26], the retardation effect of the EP interaction may favour the formation of more extended pairs. That is, due to the time-delay the second electron can take the advantage of the lattice distortion left by the first one still avoiding the direct Coulomb repulsion. In fact, increasing the EP interaction, we find that both the nearestneighbour electron-electron and electron-phonon density correlations starts to rise, while the on-site correlations remain small (cf. Fig. $7(\mathrm{~b})$ ). Consequently, we may label this state an adiabatic inter-site bipolaron. We expect that at larger values of $\varepsilon_{p}$ the short-range EP interaction overcomes the Hubbard repulsion and as a result the two electrons coalesce on a single site forming a 'self-trapped' bipolaron. Unfortunately we are unable to increase the dimension of the Hilbert space to contain a large enough number of phonons in the adiabatic very strong-coupling regime.

As already mentioned for the one-electron case, the description of the self-trapping phenomenon requires the inclusion of multi-phonon states. This is clearly displayed in Fig. 8, where we have shown the weight of the $m$-phonon state in the ground state for various EP coupling strengths. One sees immediately that the maximum of $\left|c^{m}\right|^{2}$ is rapidly shifted to larger values of $m$ as $\varepsilon_{p}$ increases. Increasing the phonon frequency at fixed $\varepsilon_{p}$, this tendency is reversed (see inset). In the extreme antiadiabatic limit $(\hbar \omega \rightarrow \infty)$ we have 
$m_{\max }=0$ and the binding disappears for $U>2 \varepsilon_{p}$.

As in the case of one electron it is interesting to look at the low-lying excitations of the inter-site bipolaron. Although we do not have a clear definition as to the momentum of this compound particle, it turns out that we indeed find a well-separated energy band if we again classify the lowest energy eigenvalues with respect to the allowed $K$-states of our finite system (see Fig. 9). The formation of the (inter-site) bipolaron band can be attributed to pronounced retardation effects [cf. the maxima in the nearest-neighbour correlation functions $C_{e l-p h}(1)$ and $C_{e l-e l}(1)$ (Fig. (7) as well as the large bipolaronic hopping amplitude $t_{b, e f f}$ (Fig. 6) at $\varepsilon_{p}=3$ ]. Surprisingly the dispersion of this 'quasiparticle' band becomes exactly like that of a free particle (with a strongly renormalized bandwidth) at $\varepsilon_{p}=$ $U / 2$, where in the standard Lang-Firsov polaron theory the effective Coulomb interaction vanishes. As the EP coupling exceeds $U / 2$, a deviation from the cosine-dispersion occurs and we expect that for $\varepsilon_{p} \gg U / 2$ an extremely narrow AHBP-band will be formed.

\section{B. Holstein $\mathbf{t}-\mathbf{J}$ model}

Now let us turn to the case, where a few dopant-induced charge carriers (holes) coupled to lattice phonons move in an antiferromagnetic correlated spin background. In 2D, this situation, frequently described by the Holstein t-J model (22) [54,24,25], is particularly interesting as it represents the basic electronic and phononic degrees of freedom in the $\mathrm{CuO}_{2}$ planes of the high- $T_{c}$ cuprates. As yet, very little is known theoretically about the interplay between EP coupling and antiferromagnetic exchange interaction in such systems. Of course, the exact diagonalization technique, as applied in the preceding section to the Holstein Hubbard model, provides reliable results for the ground-state properties of the Holstein $\mathrm{t}-\mathrm{J}$ model as well. Here, however, one usually works near half-filling, i.e., the electronic basis is very large from the outset imposing severe restrictions on the dimension of the phononic Hilbert space. Therefore we are unable to reach the extreme strong EP coupling regime especially in the adiabatic limit. In the following numerical analysis of the 
Holstein $\mathrm{t}-\mathrm{J}$ model, the exchange interaction strength is fixed to $J / t=0.4$ (which seems to be a realistic value for the high- $T_{c}$ systems).

First, let us discuss the behaviour of the effective transfer amplitude, $t_{p, e f f}=$ $E_{p, k i n}\left(\varepsilon_{p}, J\right) / E_{p, k i n}\left(\varepsilon_{p}, 0\right)$, shown in Fig. 10. Increasing the EP coupling at fixed phonon frequency $\hbar \omega=0.8$, the mobility of the hole is strongly reduced and an Holstein-type holepolaron $(\mathrm{AHP})$ is formed at about $\varepsilon_{p}^{c} \simeq 2.0$. The continuous crossover from a nearly free hole-polaron (FP) to the AHP state is similar to that observed in the 2D single-electron Holstein model, i.e., at $\varepsilon_{p} \simeq \varepsilon_{p}^{c}$ a second maximum in the phonon distribution function $\left(\left|c^{m}\right|^{2}\right)$ evolves, which, for $\varepsilon_{p} \gg \varepsilon_{p}^{c}$, becomes more pronounced and is shifted to higher phonon states. For example, we get $m_{\max } \simeq 4$ at $\varepsilon_{p}=4$ and $\hbar \omega=0.8$. The increasing importance of multiphonon states in obtaining the 'true' ground-state energy at large $\varepsilon_{p}$ becomes clearly visible in Fig. 10 by comparing the results for various phonon numbers $M$. There is, however, an important difference between the one-hole and one-electron cases which should not be underemphasized: In the single-hole Holstein t-J model antiferromagnetic spin correlations and EP interactions reinforce each other to the effect of lowering the threshold for polaronic 'self-localization'. This fact is in agreement with IMVLF-Lanczos results obtained recently by the authors [24]. As Fig. 10 illustrates, the IMVLF-Lanczos technique, which variationally takes into account inhomogeneous frozen-in displacement-field configurations as well as dynamic polaron and squeezing phenomena, describes the qualitative features of the transition from FP to AHP states and gives a reliable estimate of the renormalization of the effective transfer matrix element $t_{p, e f f}$. Moreover, the IMVLF-Lanczos method yields an excellent variational upper bound for the true ground-state energy $E_{0}$, and therefore it provides an additional educated check for the minimal number of phonons one has to take into account within the Hilbert space truncation technique.

By analogy to Eq. (8), we have calculated the corresponding hole-phonon density correlation function, $C_{h o-p h}(|i-j|)=\left\langle\Psi_{0}\left|\tilde{h}_{i} b_{j}^{\dagger} b_{j}\right| \Psi_{0}\right\rangle$, for the 2D Holstein t-J model. Figure 11 shows $C_{h o-p h}(|i-j|)$ as a function of the short-range EP interaction strength $\varepsilon_{p}$ at various phonon frequencies. The transition to the AHP state is signaled by a strong increase in 
the on-site hole-phonon correlations which are about one order in magnitude larger than the nearest-neighbour ones. This indicates that the AHP quasiparticle comprising a "quasilocalized' hole and the phonon cloud is mainly confined to a single lattice site. Increasing the phonon frequency the hole-phonon correlations are smeared out and the crossover to the small hole-polaron is shifted to larger values of the EP coupling.

Now, let us consider the two-hole case. In Fig. 12 we show the effective polaronic transfer amplitudes $t_{p, e f f}\left(N_{h}\right)$ vs EP coupling strength in the adiabatic $(\hbar \omega=0.1)$, intermediate $(\hbar \omega=0.8)$, and non-adiabatic $(\hbar \omega=3.0)$ regimes. In each case we compare the one- and two-hole results to get a feel for hole-binding effects. Remarkably we find that $t_{p, e f f}(2)$ is larger than $t_{p, e f f}(1)$ for $\varepsilon_{p} \lesssim 1$ and $\hbar \omega=0.1$, indicating a dynamical type of hole binding in the low-frequency weak-coupling regime where retardation effects become important. Indeed, the two-hole binding energy, defined as usual by $E_{B}^{2}\left(J, \varepsilon_{p}, \hbar \omega\right)=E_{0}(2)+E_{0}(0)-2 E_{0}(1)$ with respect to the Heisenberg energy $E_{0}(0)$, slightly decreases, i.e., hole binding is enhanced $\left[E_{B}^{2}(0.4,0,0)<0\right]$, as the EP interaction increases at low EP coupling strengths. In contrast, at large phonon frequencies, with increasing $\varepsilon_{p}$ we find that $E_{B}^{2}$ increases, which seems to be an indication that retardation no longer plays a role [26]. On the other hand, in the adiabatic strong-coupling regime, where the two holes become 'self-trapped' on NN sites forming a nearly immobile hole-bipolaron, we expect an even stronger reduction of $t_{p, e f f}(2)$ compared with $t_{p, e f f}(1)$ (cf. the IMVLF-Lanczos results presented in Ref. [24]. Here, a rather static type of hole binding is realized.

To substantiate this interpretation we have calculated the hole-hole density correlation function

$$
C_{h o-h o}(|i-j|)=\left\langle\Psi_{0}\left(\varepsilon_{p}, J\right)\left|\tilde{h}_{i} \tilde{h}_{j}\right| \Psi_{0}\left(\varepsilon_{p}, J\right)\right\rangle
$$

in the 2D Holstein $\mathrm{t}-\mathrm{J}$ model. Note that $C_{h o-h o}(|i-j|)$ provides an even more reliable test for the occurrence of hole binding than the binding energy $E_{B}^{2}$ [55]. Indeed, when calculating $E_{B}^{2}$, we are comparing states with different quantum numbers, specifically with different $S$ and $S^{z}$. In Fig. 13 we present results for the non-equivalent hole-hole pair 
correlation functions in the ground state of the Holstein $\mathrm{t}-\mathrm{J}$ model with two holes. In the weak-coupling region the hole-density correlation function becomes maximum at the largest distance of the ten-site lattice, while in the intermediate EP coupling regime the preference is on NNN pairs. As expected, increasing further the EP interaction strength, the maximum in $C_{h o-h o}(|i-j|)$ is shifted to the shortest possible distance (remember that double occupancy is strictly forbidden), indicating hole-hole attraction. The behaviour of $C_{h o-h o}(|i-j|)$ is found to be qualitatively similar for higher (lower) phonon frequencies (see inset), except that the crossings of different hole-hole correlation functions occur at larger (smaller) values of $\varepsilon_{p}$. In essence, our results clearly indicate that hole-bipolarons could be formed in the Holstein $\mathrm{t}-\mathrm{J}$ model at large EP coupling.

\section{CONCLUSIONS}

To summarize, in this paper we have studied the problem of (hole--) bi-/polaron formation in the Holstein Hubbard/t-J model by means of direct Lanczos diagonalization using a truncation method of the phononic Hilbert space. Compared with previous treatments of the Holstein (Hubbard) model on very small clusters, we are able to analyze large enough systems in order to discuss polaron and bipolaron band formation, which has been a subject of recent controversy [1,9]. Our main results are the following.

(i) In the case of a single electron coupled to Einstein phonons (Holstein model), we confirm that the rather 'sharp' transition from a 'delocalized' nearly free polaron (FP) [or a large polaron (LP) in 1D] to a 'quasi-localized' Holstein polaron (AHP) in the adiabatic regime and the very smooth transition to a Lang-Firsov-type polaron (NLFP) in the non-adiabatic regime are both continuous. In agreement with recent exact results [27,29,30], we observe no ground-state level crossings or any non-analyticities as the EP coupling increases. We point out that in the one-dimensional weak-coupling case a large-size polaron is formed at any finite EP coupling. In the strong-coupling regime, the AHP state is characterized by pronounced on-site electron-phonon cor- 
relations making the quasiparticle susceptible to 'self-trapping'. Most notably, the formation of an adiabatic Holstein polaron is accompanied by a shift of the maximum in the phonon distribution function to higher phonon states, which seems to be an intrinsic feature of the 'self-trapping' transition. By contrast, the non-adiabatic NLFP ground state is basically a zero-phonon state.

(ii) By calculating the spectral properties of a single electron, we have found convincing evidence for the formation of a well separated narrow polaron band in both the adiabatic and non-adiabatic strong-coupling regimes. In addition to the expected band-narrowing we also found a deviation from the 'cosine'-dispersion away from the adiabatic and antiadiabatic limits. Although the 'coherent' bandwidth, deduced from our finite-lattice ED data, becomes extremely small in the adiabatic strong-coupling case (polaronic band collapse), we believe that the AHP does not lose its phase coherence and can move itinerantly.

(iii) Investigating the two-particle problem in terms of the 1D Holstein model, we could clearly identify the transition from a extended (large) bipolaron to a 'quasi-localized' (on-site) bipolaron (AHBP) as the EP interaction strength increases. Stabilizing a two-polaron state in the weak EP coupling regime by taking into account the on-site Coulomb repulsion (Holstein Hubbard model), we found a transition to an inter-site bipolaron at about $\varepsilon_{p} \simeq U / 2$. It is worth emphasizing that this inter-site bipolaron appears to have a dispersion that resembles very closely the cosine-dispersion of a noninteracting particle with a renormalized bandwidth. If the EP coupling is further enhanced $\left(\varepsilon_{p} \gg U / 2, \hbar \omega, t\right)$, a second transition to a 'self-trapped' on-site AHBP will occur [51].

(iv) Analyzing the hole-polaron formation in the framework of the 2D Holstein $\mathrm{t}-\mathrm{J}$ model, we found that the critical EP coupling for the polaron transition is substantially reduced due to 'prelocalization' of the doped charge carriers in the antiferromagnetic spin 
background. Therefore we suggest that polaronic effects are of special importance in (low-dimensional) strongly correlated narrow-band systems like the nickelates and high- $T_{c}$ cuprates.

(v) Regarding ground--state properties of the Holstein t-J model in the two-hole sector, a detailed study of the hole-hole correlation functions and the two-hole binding energy was carried out, yielding strong evidence for an enhanced hole attraction and the formation of hole-bipolarons as a dynamical effect of the EP interaction.

Of course, the exact results presented in this paper hold for the Holstein Hubbard (Holstein t-J) model with one and two electrons (holes) on finite 1D (2D) systems, i.e., we are not prepared to prove any rigorous statements about the thermodynamic limit here. However, we believe that our main conclusions (i)-(v), in particular the existence of well-separated polaronic and bipolaronic quasiparticle bands even in the adiabatic strong-coupling regime, will survive in the infinite system.

\section{ACKNOWLEDGEMENTS}

The computations were performed on a CM5 of the GMD (St. Austin). We thank D. Ihle and E. Salje for interesting and helpful discussions and J. Stolze for a critical reading of the manuscript. 


\section{REFERENCES}

[1] Y. Bar-Yam, T. Egami, J. M. de Leon, and A. R. Bishop, Lattice Effects in High-T Superconductors, World Scientific, (Singapore 1992).

[2] H. Rietschel, J. Low Temp. Phys. 95, 293 (1994).

[3] M. Grilli and C. Castellani, Phys. Rev. B 50, 16880 (1994).

[4] J. Ranninger, Z. Phys. B 84, 167 (1991).

[5] A. S. Alexandrov and A. B. Krebs, Usp. Fiz. Nauk 162, 1 (1992).

[6] D. Emin, Phys. Rev. B 45, 5525 (1992).

[7] X.-X. Bi and P. C. Eklund, Phys. Rev. Lett. 70, 2625 (1993).

[8] A. S. Alexandrov and N. F. Mott, Int. J. Mod. Phys. B 8, 2075 (1994).

[9] E. K. H. Salje, A. S. Alexandrov, and W. Y. Liang, Polarons and Bipolarons in High Temperature Superconductors and Related Materials, Cambridge Univ. Press, (Cambridge 1995).

[10] C. H. Chen, S.-W. Cheong, and A. S. Cooper, Phys. Rev. Lett. 71, 2461 (1993).

[11] D. Mihailović, C. M. Foster, K. Voss, and A. J. Heeger, Phys. Rev. B 42, 7989 (1990).

[12] P. Calvani, Solid State Commun. 91, 113 (1994).

[13] J. P. Falck, A. Levy, M. A. Kastner, and R. J. Birgenau, Phys. Rev. B 48, 4043 (1993).

[14] J. Ranninger and U. Thibblin, Phys. Rev. B 45, 7730 (1992).

[15] J. Mustre de Leon, I. Batistić, A. R. Bishop, S. D. Conradson, and S. A. Trugman, Phys. Rev. Lett. 68, 3236 (1992).

[16] J. Zhong and H.-B. Schüttler, Phys. Rev. Lett. 69, 1600 (1992).

[17] J. Ranninger, Phys. Rev. B 48, 13166 (1993).

[18] A. S. Alexandrov, V. V. Kabanov, and D. K. Ray, Phys. Rev. B 49, 9915 (1994).

[19] H.-B. Schüttler, K. Yonemitsu, and J. Zhong, Journal of Superconductivity 8, No. 1 (1995).

[20] H. Fehske et al., Z. Phys. 94, 91 (1994).

[21] J. Lorenzana and A. Dobry, Phys. Rev. B 50, 16094 (1994). 
[22] H. Röder, H. Fehske, and R. N. Silver, Europhys. Lett. 28, 257 (1994).

[23] J. Zaanen and P. B. Littlewood, Phys. Rev. B 50, 7222 (1994).

[24] H. Fehske, H. Röder, G. Wellein, and A. Mistriotis, Phys. Rev. B 51, 16582 (1995).

[25] A. Dobry, A. Greco, S. Koval, and J. Riera, cond-mat/9505028, preprint (1995).

[26] F. Marsiglio, Physica C 244, 21 (1995).

[27] H. Löwen, Phys. Rev. B 37, 8661 (1988).

[28] B. Gerlach and H. Löwen, Rev. Mod. Phys. 63, 63 (1991).

[29] S. Ciuchi, F. de Pasquale, and D. Feinberg, EuroPhys. Lett. 30, 151 (1995).

[30] J. K. Freericks and E. H. Lieb, Phys. Rev. B 51, 2812 (1995).

[31] A. B. Migdal, Sov. Phys. JETP 7, 999 (1958).

[32] G. M. Eliashberg, Sov. Phys. JETP 11, 696 (1960).

[33] T. Holstein, Ann. Phys. 8, 325 (1959).

[34] I. G. Lang and Y. A. Firsov, Zh. Eksp. Teor. Fiz. 43, 1843 (1962).

[35] H. Zheng, D. Feinberg, and M. Avignon, Phys. Rev. B 39, 9405 (1989).

[36] D. Feinberg, S. Ciuchi, and F. de Pasquale, Int. J. Mod. Phys. B 4, 1317 (1990).

[37] U. Trapper, H. Fehske, M. Deeg, and H. Büttner, Z. Phys. B 93, 465 (1993).

[38] F. Marsiglio, Phys. Lett. A 180, 280 (1993).

[39] H. D. Raedt and A. Lagendijk, Phys. Rev. Lett. 49, 1522 (1982).

[40] H. D. Raedt and A. Lagendijk, Phys. Rev. B 27, 6097 (1983).

[41] J. E. Hirsch and E. Fradkin, Phys. Rev. Lett. 49, 402 (1982).

[42] R. M. Noack and D. J. Scalapino, Phys. Rev. B 47, 305 (1993).

[43] J. E. G. P. Niyaz, R. T. Scalettar, and C. Y. Fong, Phys. Rev. B 48, 16011 (1993).

[44] H. Schüttler, J. Zhong, and A. J. Fedro, Physica C 185-189 (1991).

[45] H. J. Schulz and T. A. Ziman, EuroPhys. Lett. 18, 355 (1992).

[46] H. Fehske, H. Röder, A. Mistriotis, and H. Büttner, J. Phys. Condens. Matter 5, 3635 (1993). 
[47] H. Röder, H. Fehske, and H. Büttner, Phys. Rev. B 47 (1993).

[48] Y. Inada and C. Ishii, J. Phys. Soc. Jpn. 59, 612 (1990).

[49] D. Emin and T. Holstein, Phys. Rev. Lett. 36, 323 (1976).

[50] The density-displacement correlation function has been recently discussed by Marsiglio [26] for a single electron on a four-site ring (with 40 phonons) and on a eight-site lattice, but with a fewer total number of phonons (10); so the author was unable to check finite-size and truncated Hilbert space effects in the "highly polaronic" strong-coupling regime $(\hbar \omega \ll t)$.

[51] G. Wellein, Master's thesis, University of Bayreuth, (1994).

[52] R. N. Silver and H. Röder, Int. J. Mod. Phys. C 5, 935 (1994).

[53] D. Ihle and H. Fehske, J. Phys. A 28, L275 (1995).

[54] A. N. Das, J. Konior, and D. K. Ray, Physica C 170, 215 (1990).

[55] J. Bonča, P. Prelovšek, and I. Sega, Phys. Rev. B 39, 7074 (1989). 


\section{FIGURES}

FIG. 1. Ground-state energy $E_{0}$ and weight of the $m$-phonon states $\left|c^{m}\right|^{2}$ (inset) as a function of the maximal number of phonons $M$ for the 1D single-electron Holstein model on a four-site lattice. The model parameters are: $\varepsilon_{p}=6.0, \hbar \omega=0.4$ (all energies are measured in units of $t$ ).

FIG. 2. Effective hopping amplitude, $t_{p, \text { eff }}$ vs $\varepsilon_{p}$, for a single electron on a ten-site lattice described by the Holstein model.

FIG. 3. Electron-phonon density correlation function $C_{e l-p h}(|i-j|)$ as a function of the distance $i-j$ and EP coupling $\varepsilon_{p}$ (inset) at $\hbar \omega=0.4$. The results are given for the single-electron Holstein model on a eight-site chain.

FIG. 4. Single-electron band dispersion $E_{K}-E_{0}$ at $\varepsilon_{p}=3$ in units of the coherent bandwidth $\Delta E$. The inset shows the distribution of the eigenvalues at $\hbar \omega=0.4$, where the horizontal axis counts the eigenvalues sorted by magnitude and the vertical axis gives their absolute values $E_{n}:=E_{n}^{(t o t)}-N \hbar \omega / 2$. All results are given for the eight-site chain with $M=18$ phonons.

FIG. 5. Band dispersion $E(K)$ of a single electron described by the Holstein model on $1 \mathrm{D}$ rings with $N$ sites, where $\varepsilon_{p}=3.0$ and $\hbar \omega=0.4$. The insets show the low-energy part of the one-particle spectral function $A_{K}(\mathrm{E})$ taken at the $K$-values indicated by arrows. The dotted line corresponds to the dispersion of a free particle with a renormalized bandwidth.

FIG. 6. The effective polaron and bipolaron transfer amplitudes, $t_{p, \text { eff }}$ and $t_{b, e f f}$, are shown as a function of the EP coupling strength $\varepsilon_{p}$ in (a) and (b), respectively. The results are given for two electrons on the eight-site chain with $M=21$ phonons using periodic boundary conditions.

FIG. 7. Electron-electron [electron-phonon] correlation function $C_{e l-e l}\left[C_{e l-p h}\right.$ (inset)] vs $\varepsilon_{p}$ at $\hbar \omega=0.4$ for $U=0(\mathrm{a})$ and $U=6(\mathrm{~b})$.

FIG. 8. Weight of the $m$-phonon state in the two-electron ground state of the Holstein Hubbard model, where $U=6$ and $\hbar \omega=0.4[\hbar \omega=3$ (inset)]. 
FIG. 9. The lowest eigenvalues $E_{K}$ for the two-electron case on the eight-site chain, where $\hbar \omega=0.4$ and $U=6$. The energy band is given in units of the coherent bandwidth $\Delta E=0.0481$, 0.0217 , and 0.0190 for $\varepsilon_{p}=2.5,2.75$, and 3.0 , respectively.

FIG. 10. Effective hopping amplitude $t_{p, \text { eff }}$ and ground-state energy $E_{0}$ (inset) as a function of EP coupling strength $\varepsilon_{p}$ for the 2D single-hole Holstein t-J model at $J=0.4$ and $\hbar \omega=0.8$. Exact results for the ten-site square with different numbers of phonons $M$ are compared with approximative IMVLF-Lanczos data 24]. For further explanation see text.

FIG. 11. Dependence of the on-site and nearest-neighbour (inset) hole-phonon correlation function $C_{h o-p h}(|i-j|)$ on the EP coupling $\varepsilon_{p}$ and phonon frequency $\hbar \omega$ at $J=0.4$. Results are given for a single hole on a ten-site lattice with at most $M=12$ phonons.

FIG. 12. Effective transfer amplitudes, $t_{p, \text { eff }}\left(N_{h}\right) v s \varepsilon_{p}$, are compared for the one- and two-hole cases at various phonon frequencies, where $J=0.4$ and $M=12$.

FIG. 13. Hole-hole density correlation function $C_{h o-h o}(|i-j|)$ of the 2D Holstein t-J model shown as a function of the EP coupling $\varepsilon_{p}$ for $J=0.4$ at $\hbar \omega=0.8$ and $\hbar \omega=0.1$ (inset), where the numbers $1-3$ label the non-equivalent distances $\left|\vec{R}_{i}-\vec{R}_{j}\right| / a=1, \sqrt{2}$, and $\sqrt{5}$ between NN, next NN, and third NN sites on a ten-site lattice with $M=12$ phonons, respectively. 


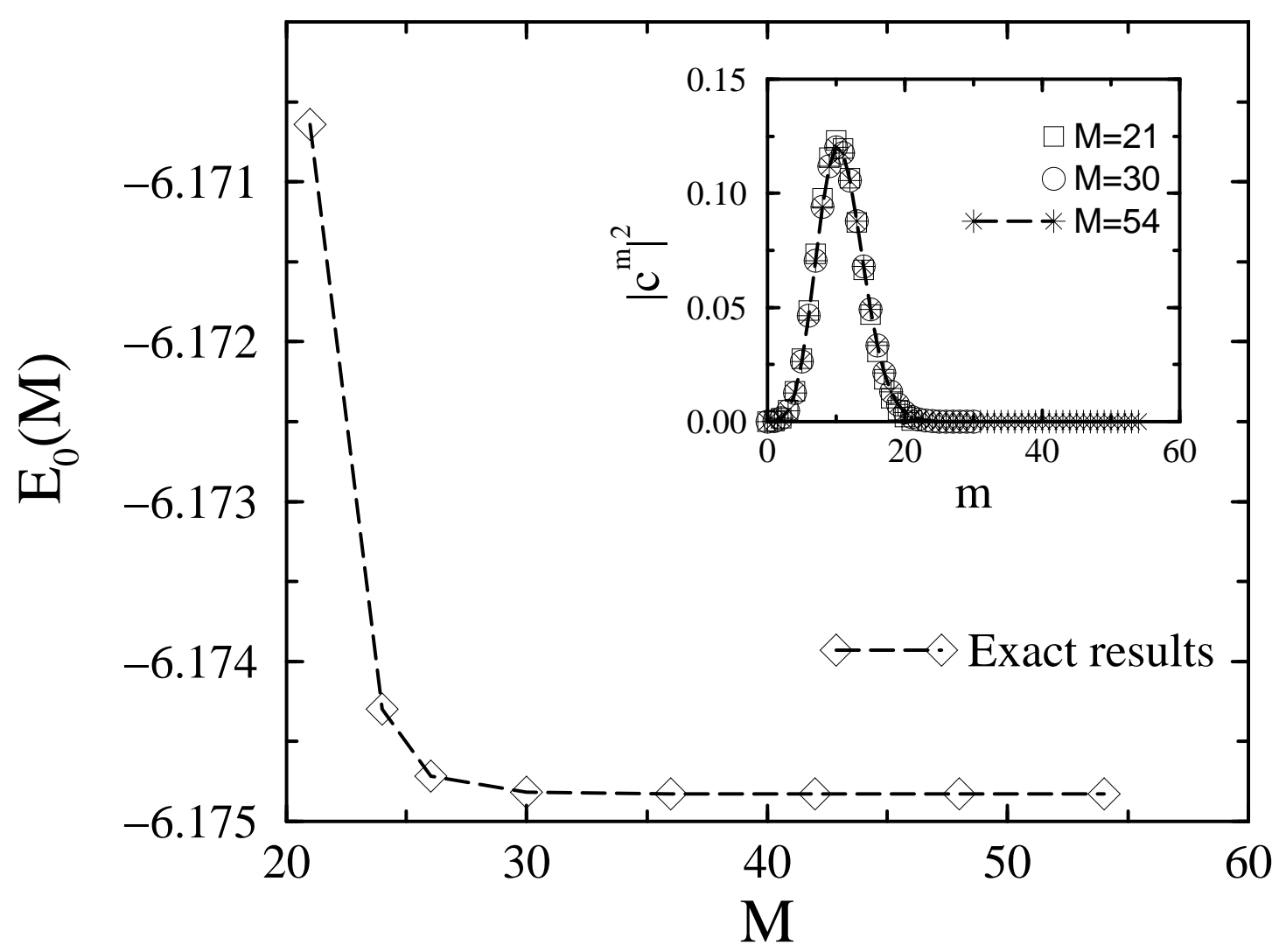

Figure 1: 


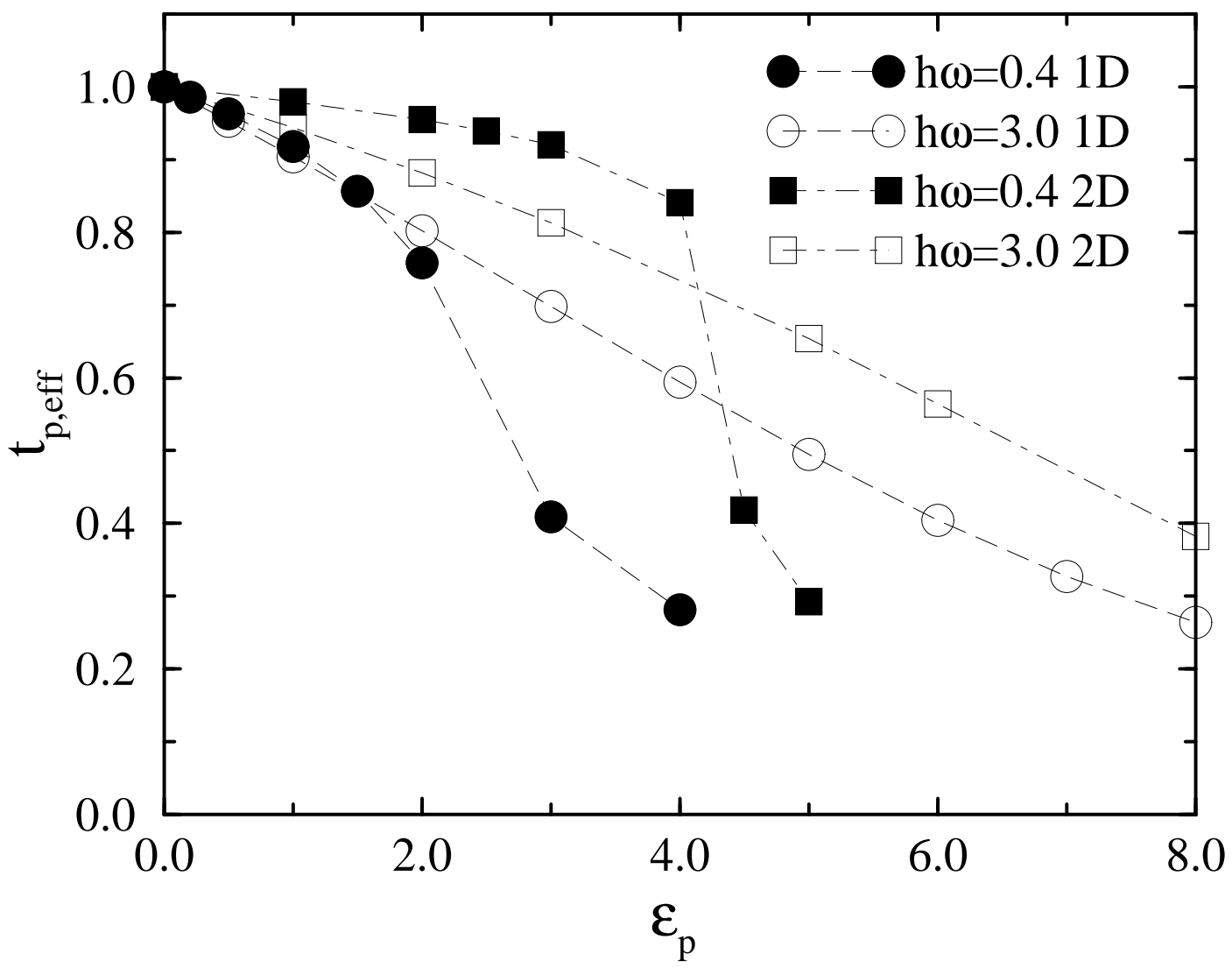

Figure 2: 


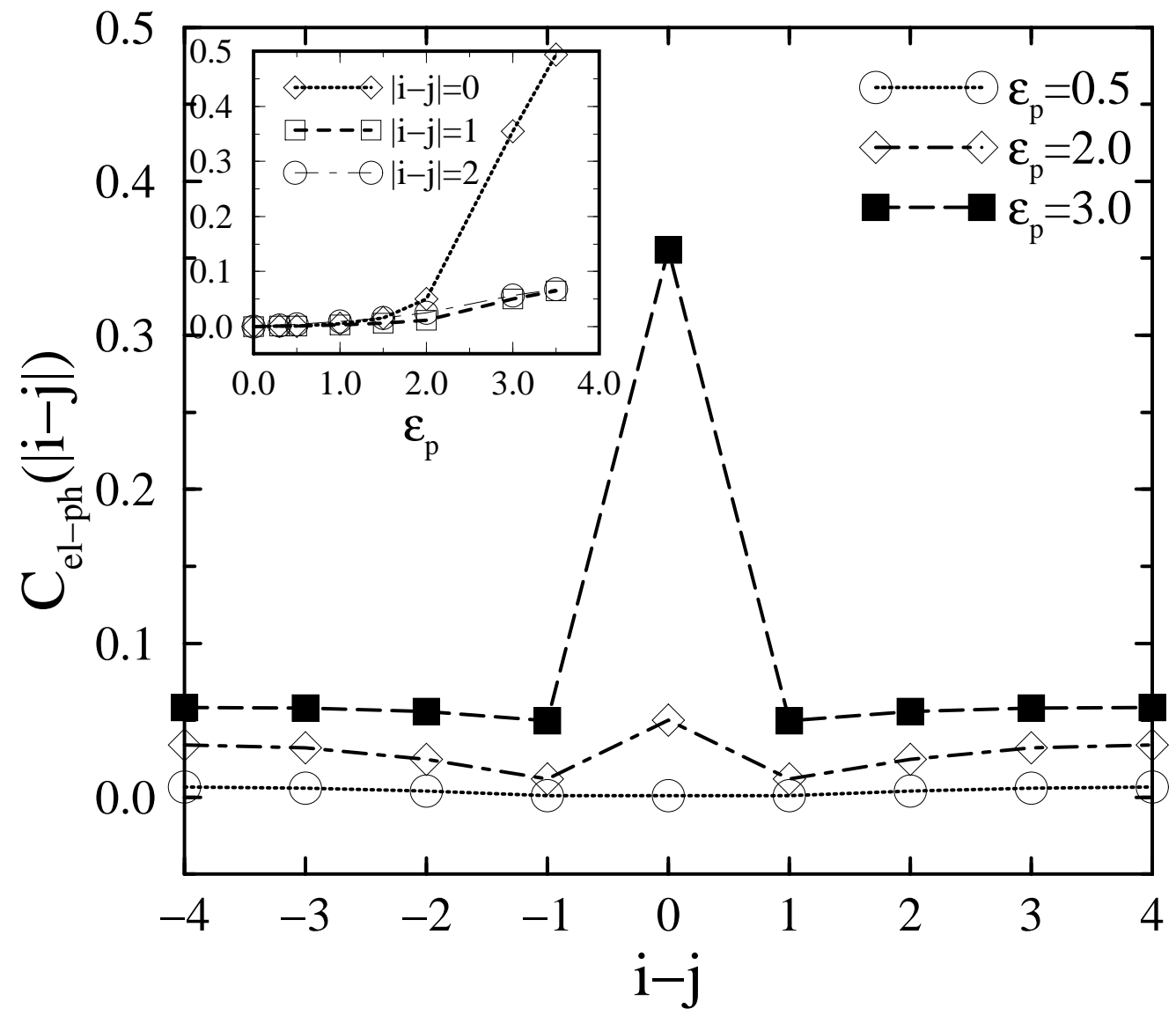

Figure 3: 


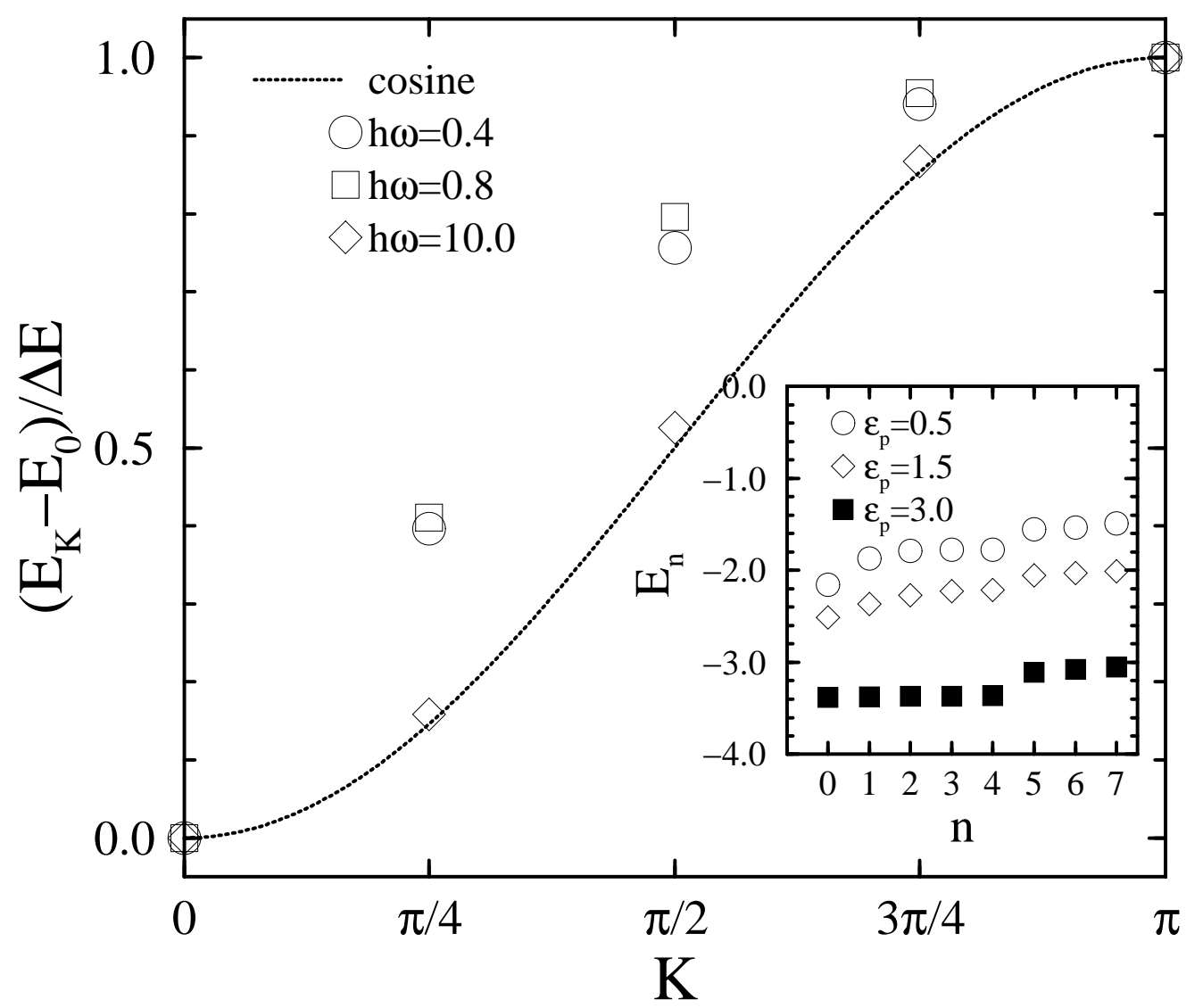

Figure 4: 


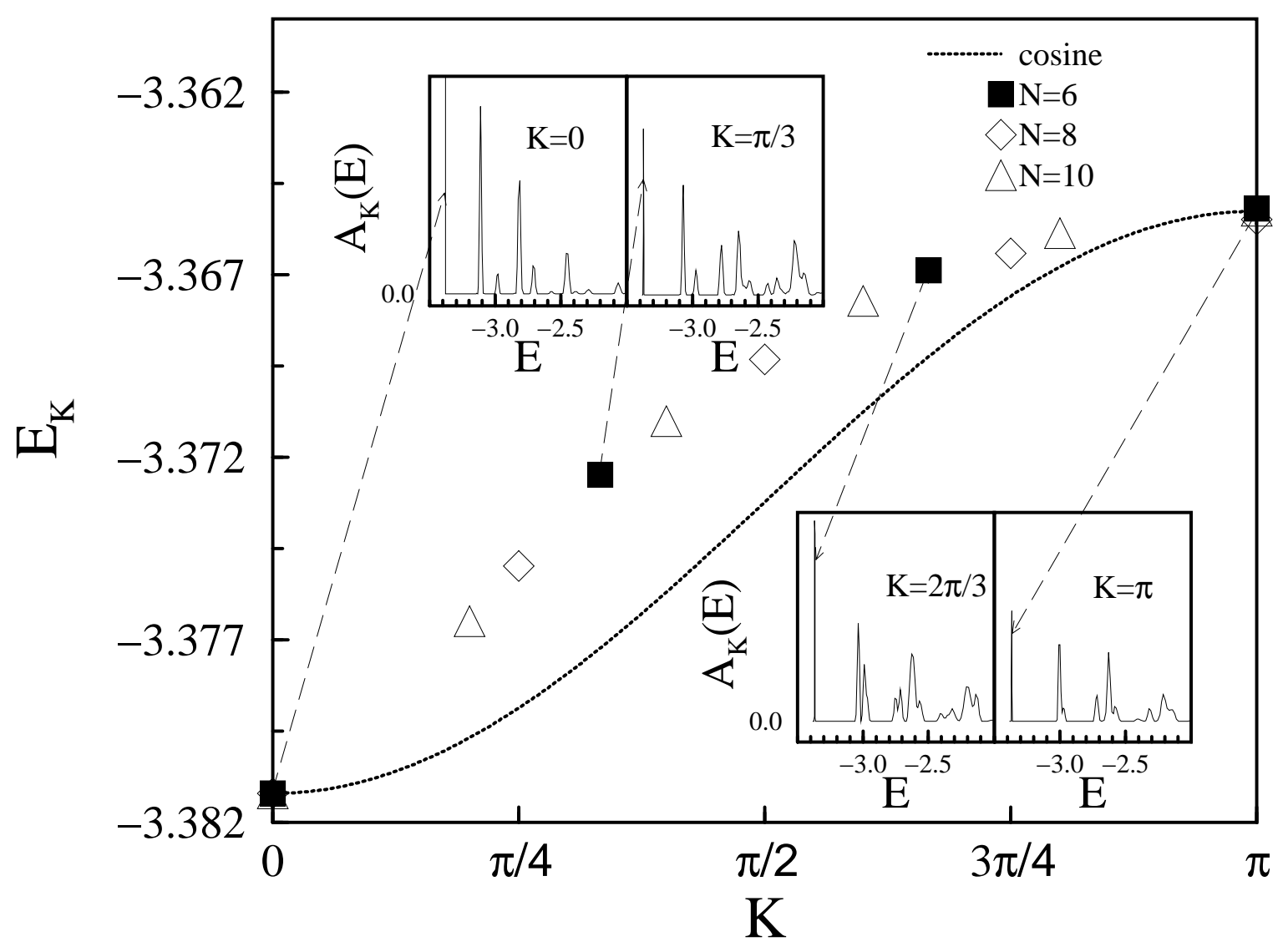

Figure 5: 

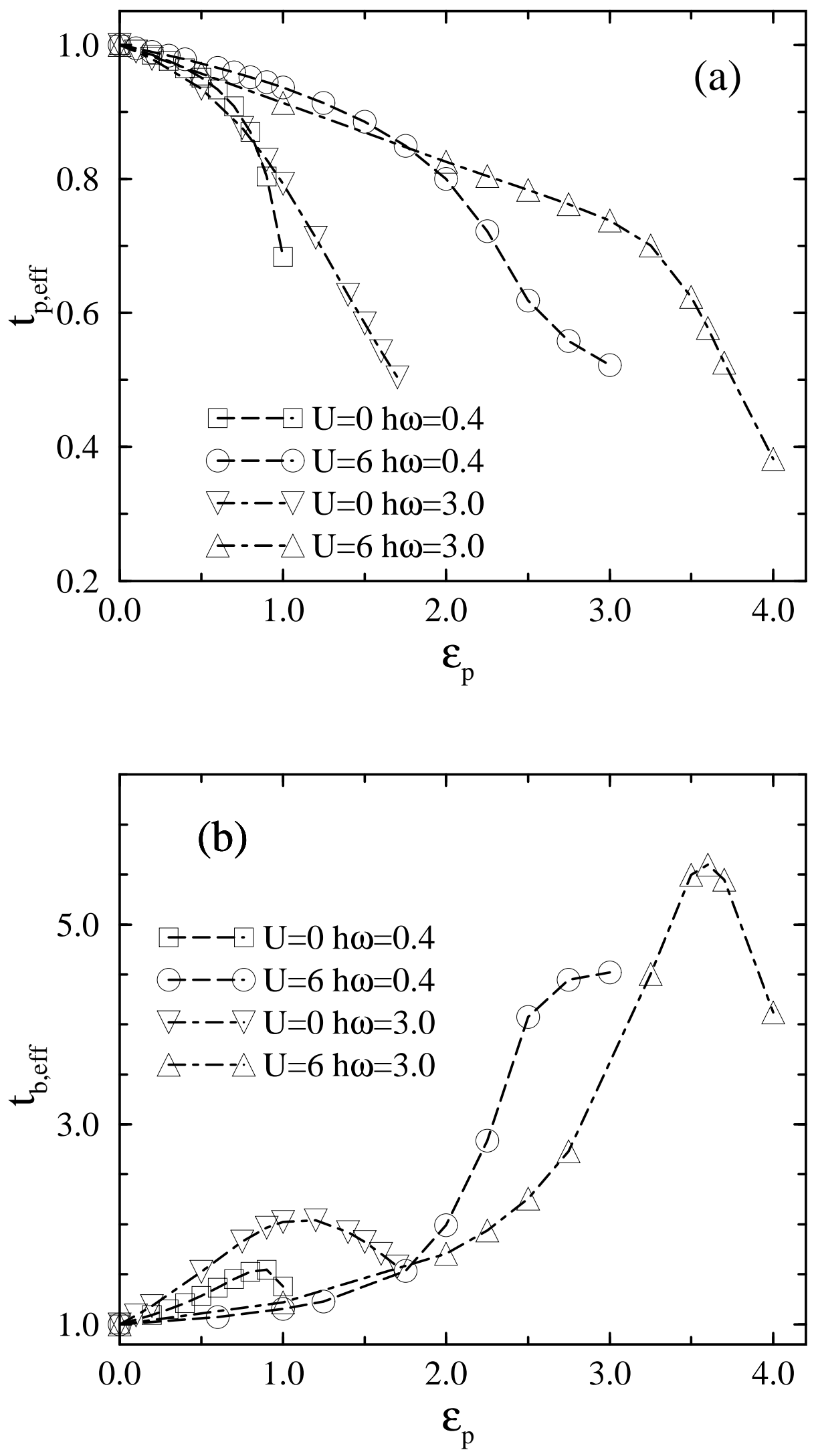

Figure 6: 

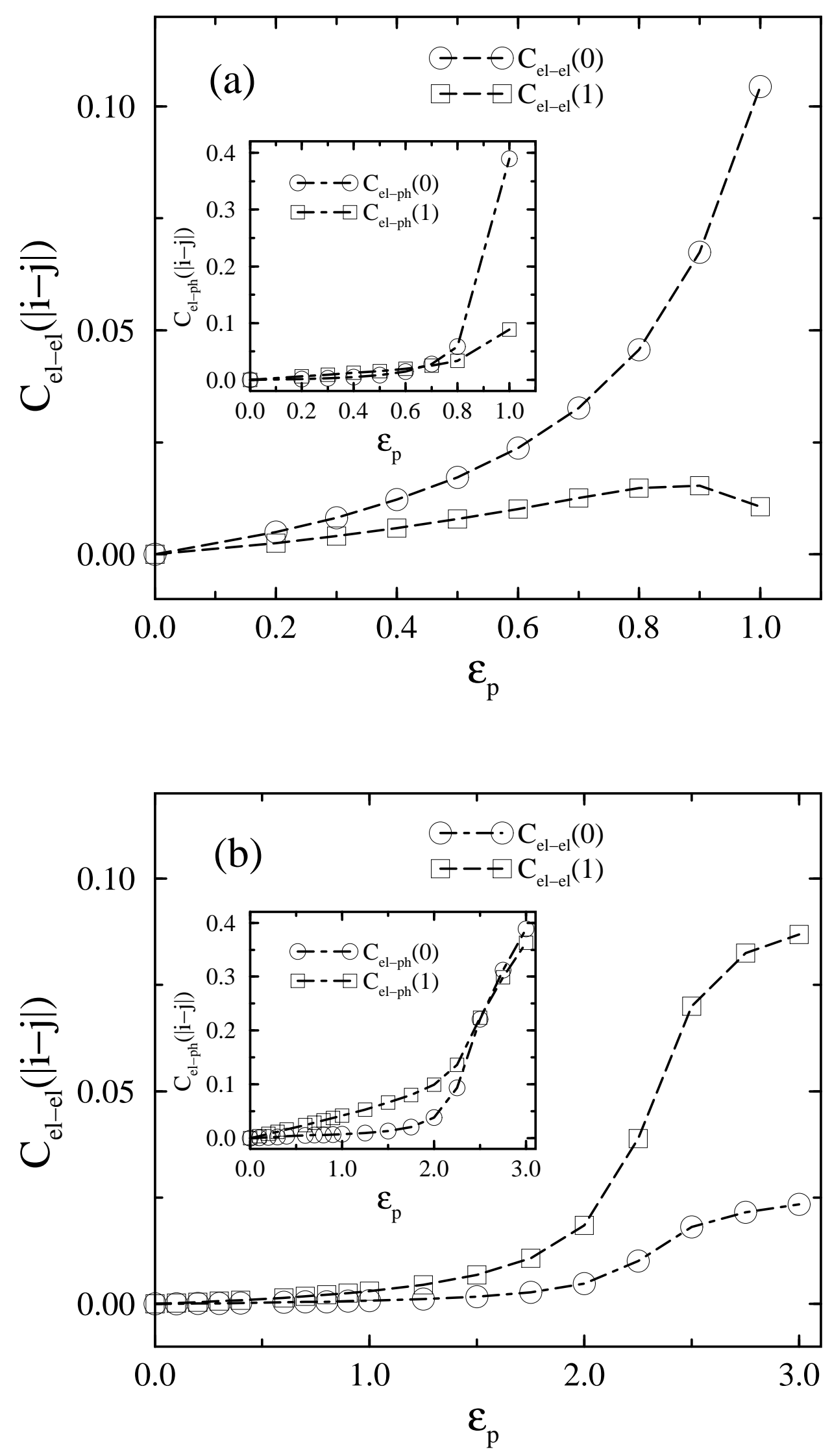

Figure 7: 


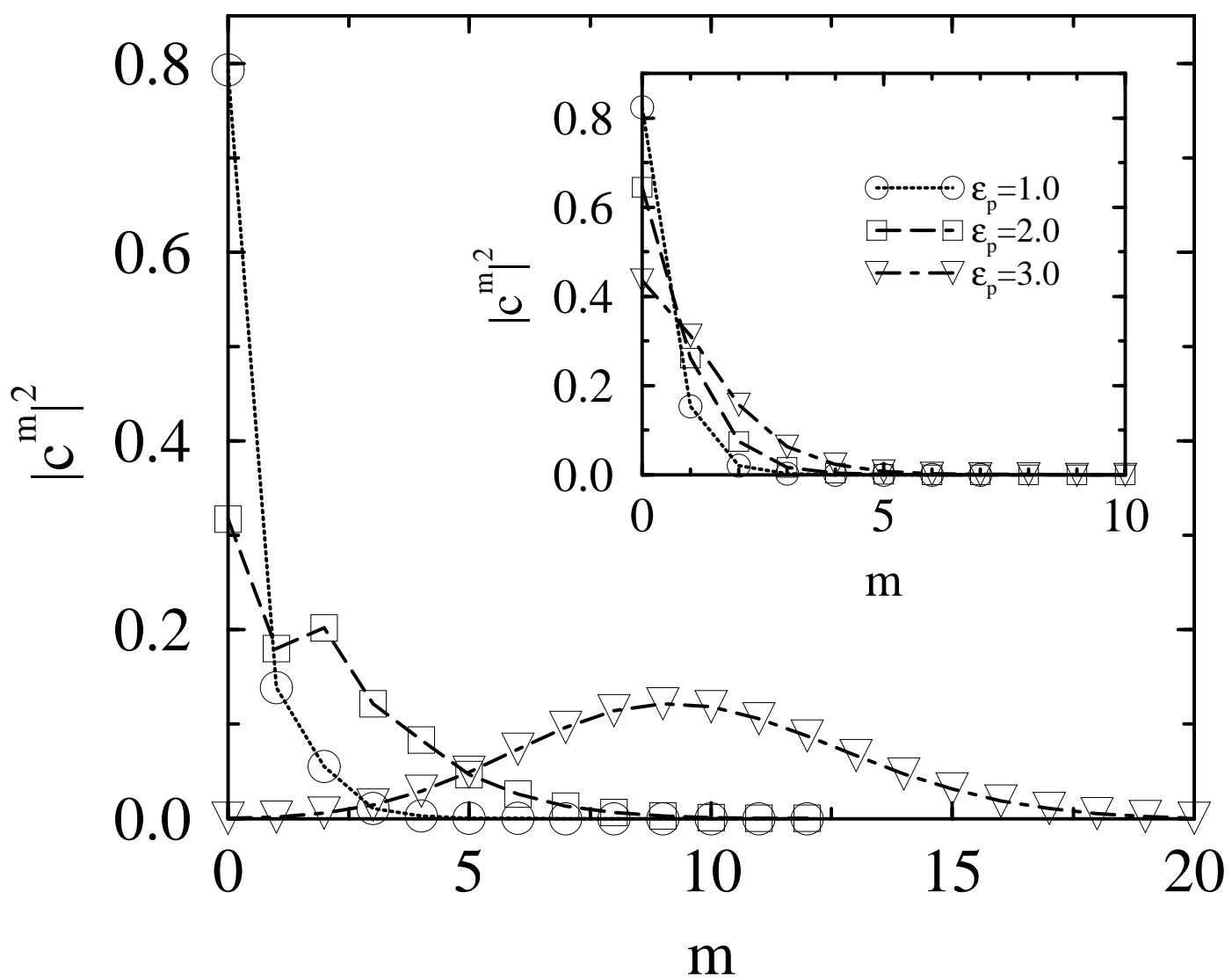

Figure 8: 


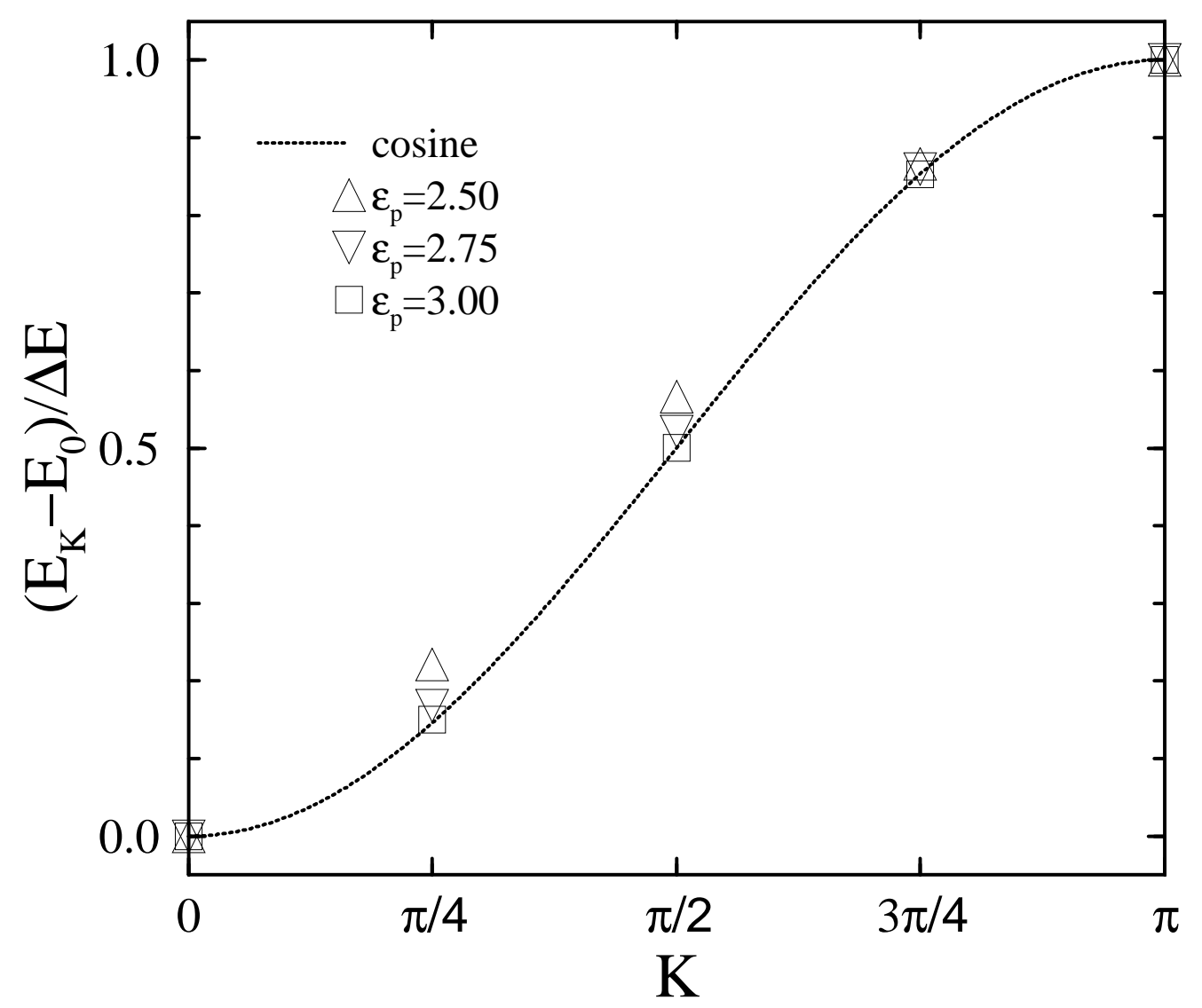

Figure 9: 


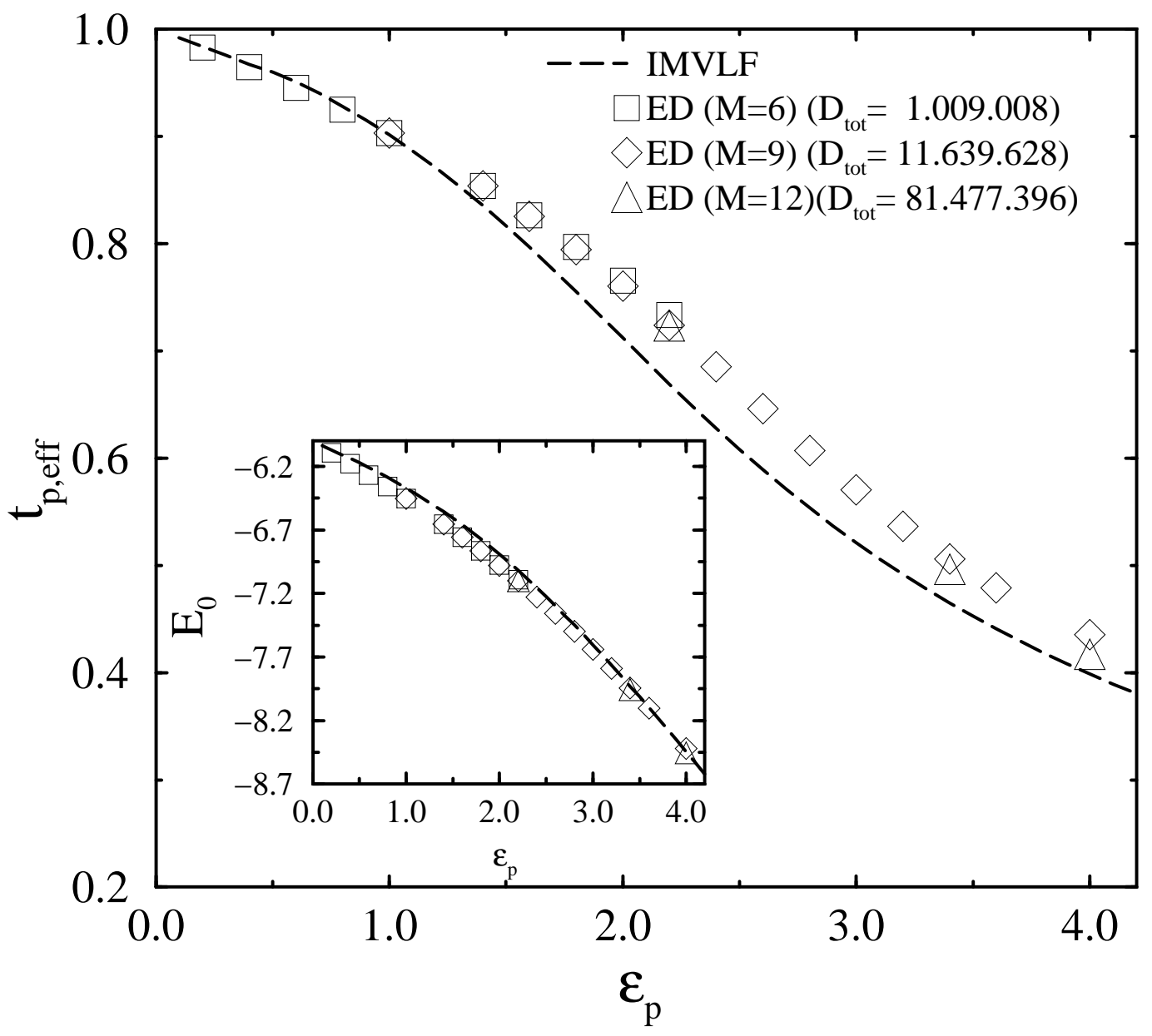

Figure 10: 


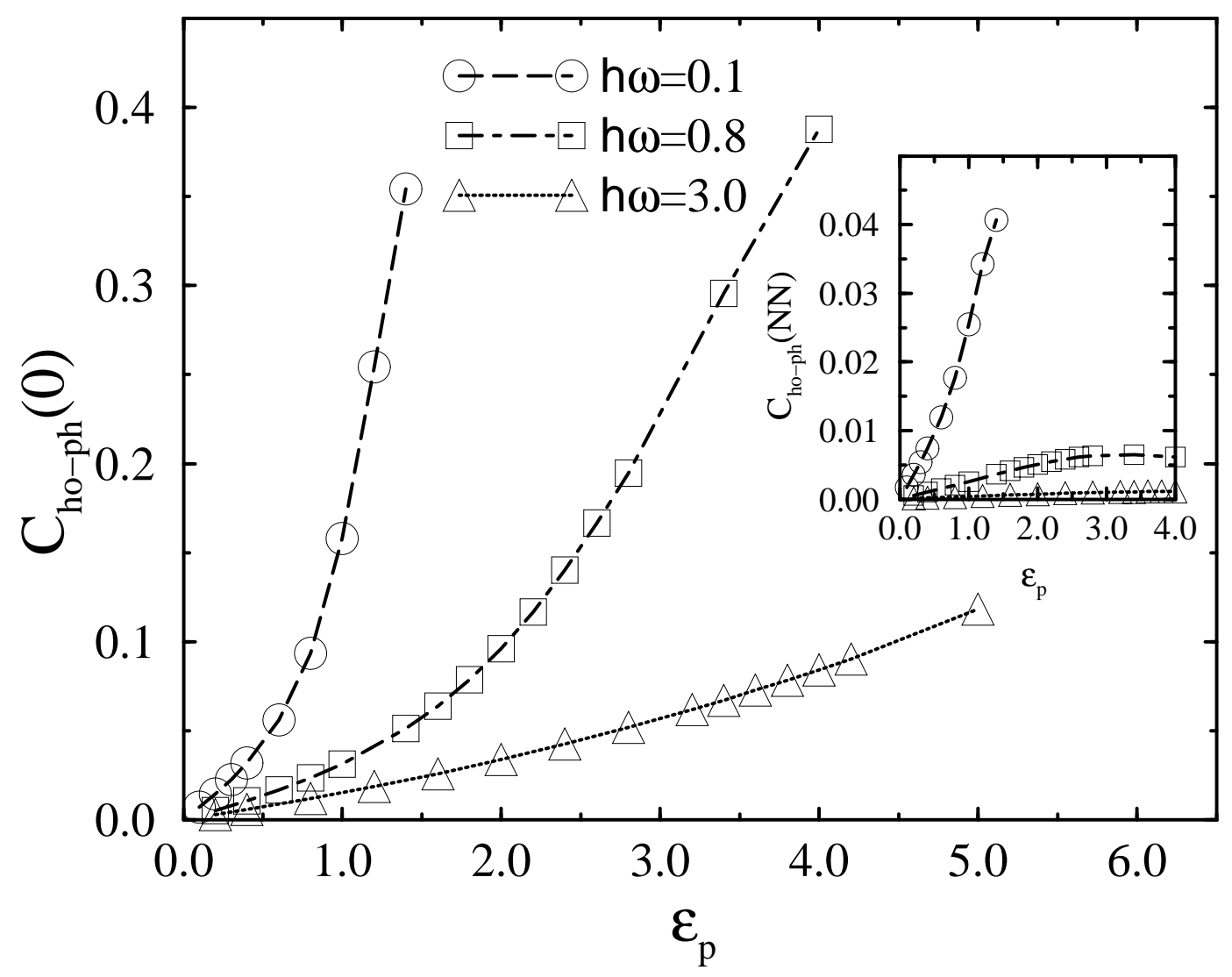

Figure 11: 


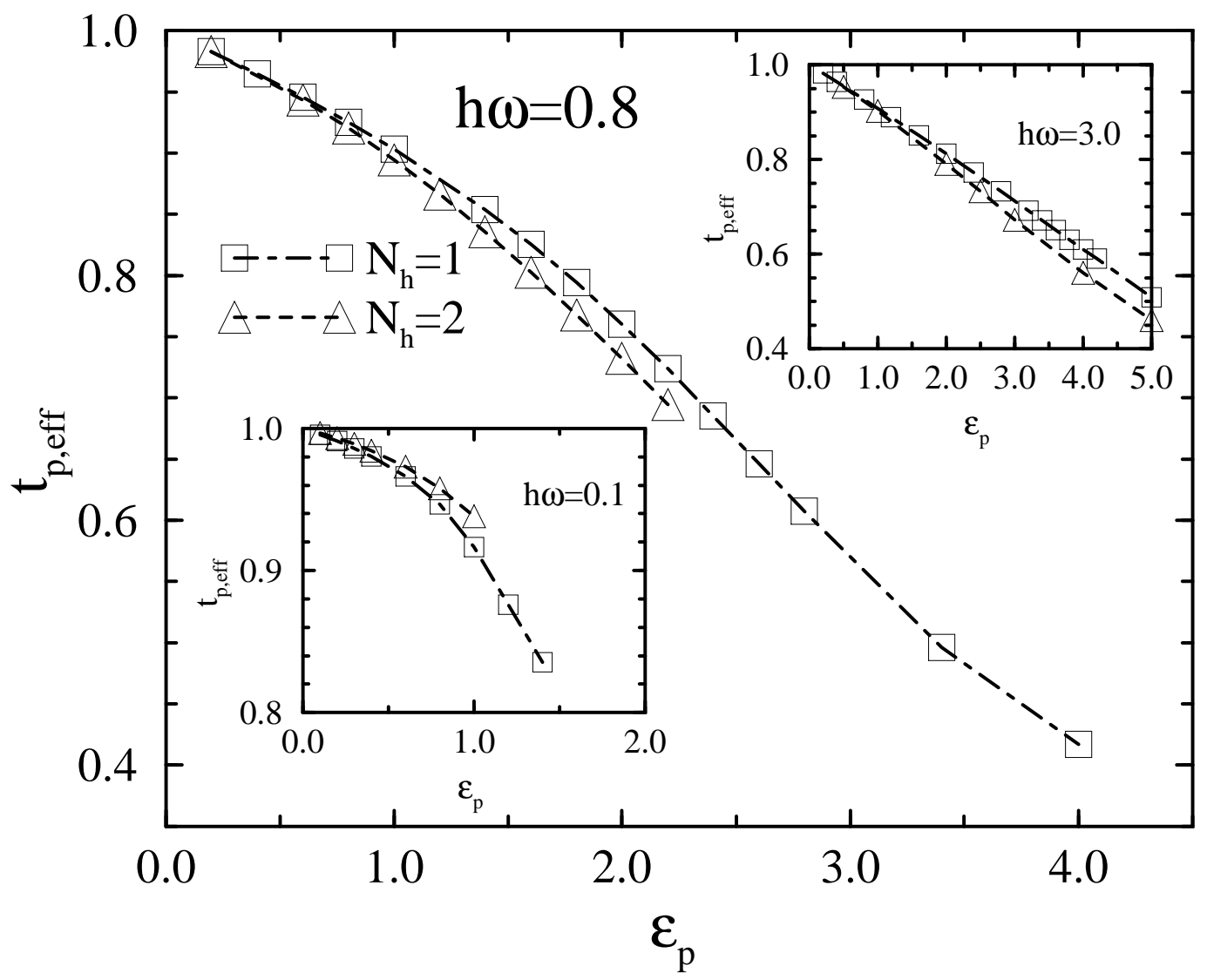

Figure 12: 


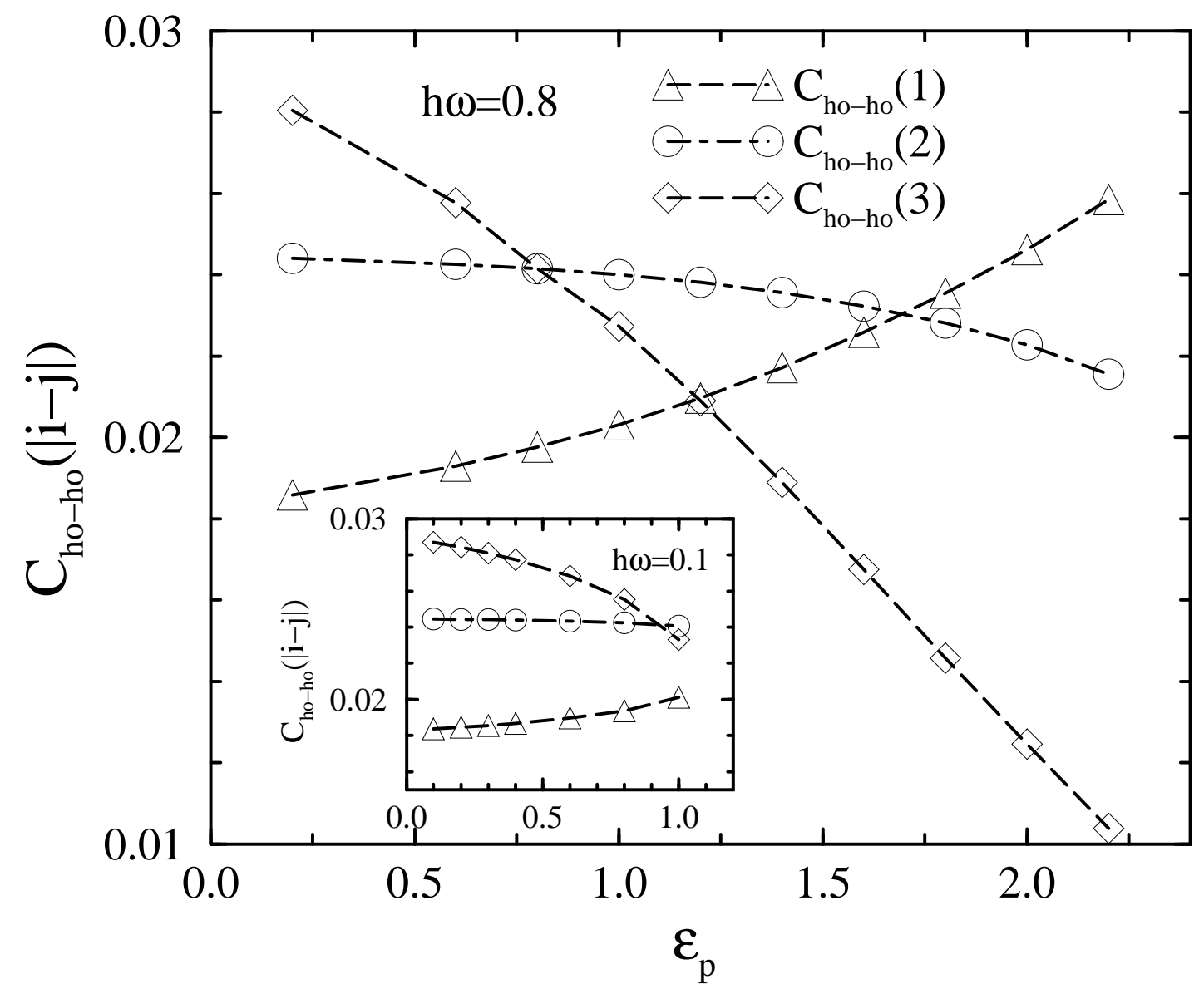

Figure 13: 